\title{
Growth and magnetotransport study of thin ferromagnetic $\mathrm{CrO}_{2}$ films
}

\author{
M Rabe ${ }^{1}$, J Pommer ${ }^{1}$, K Samm ${ }^{1}$, B Özyilmaz ${ }^{1}$, C König ${ }^{1}$, M Fraune ${ }^{1}$, \\ U Rüdiger ${ }^{1}$, G Güntherodt ${ }^{1}$, S Senz ${ }^{2}$ and D Hesse ${ }^{2}$ \\ ${ }^{1}$ II. Physikalisches Institut, Rheinisch-Westfälische Technische Hochschule Aachen, \\ D-52056 Aachen, Germany \\ ${ }^{2}$ Max-Planck-Institut für Mikrostrukturphysik, Weinberg 2, D-06120 Halle/Saale, Germany
}

\begin{abstract}
Highly $a$-axis-textured $\mathrm{CrO}_{2}$ films have been deposited on $\mathrm{Al}_{2} \mathrm{O}_{3}(0001)$ and on isostructural $\mathrm{TiO}_{2}(100)$ substrates by a chemical vapour deposition technique. For $\mathrm{Al}_{2} \mathrm{O}_{3}$ substrates a columnar growth of $\mathrm{CrO}_{2}(010)$ on an initial $\mathrm{Cr}_{2} \mathrm{O}_{3}(0001)$ layer has been found in transmission electron microscopy as well as in $\mathrm{x}$-ray diffraction investigations. The sixfold in-plane symmetry of a (0001)-oriented $\mathrm{Cr}_{2} \mathrm{O}_{3}$ initial layer leads to three equivalent in-plane orientations of the $\mathrm{CrO}_{2}$ unit cell as confirmed by electron diffraction and scanning electron microscopy. The growth can be understood by a simple model of the in-plane symmetries of the $\mathrm{Al}_{2} \mathrm{O}_{3}(0001), \mathrm{Cr}_{2} \mathrm{O}_{3}(0001)$, and $\mathrm{CrO}_{2}(010)$ lattices. The growth on $\mathrm{TiO}_{2}(100)$ substrates leads to (100)-oriented $\mathrm{CrO}_{2}$ films of higher crystalline quality than the ones grown on $\mathrm{Al}_{2} \mathrm{O}_{3}(0001)$. Transmission electron microscope images show growth of $\mathrm{CrO}_{2}(100)$ directly on the $\mathrm{TiO}_{2}(100)$ substrates and no significant $\mathrm{Cr}_{2} \mathrm{O}_{3}$ inclusions within the $\mathrm{CrO}_{2}(100)$ layer. All contributions to the magnetoresistance (MR) due to anisotropic MR, Lorentz MR, spin disorder, and intergrain tunnelling MR have been determined and partly correlated with the crystalline properties of the samples investigated. For films of both types the intrinsic linear contribution to the high-field MR does not depend on the crystalline quality of the films and supports the suggested intrinsic doubleexchange mechanism for $\mathrm{CrO}_{2}$.
\end{abstract}

\section{Introduction}

The theoretically predicted $100 \%$ spin polarization at the Fermi energy $E_{F}$ of $\mathrm{CrO}_{2}[1]$ makes it a promising material for magnetoelectronic devices. According to Jullière's model [2] the magnetoresistance (MR) of ferromagnet/insulator/ferromagnet tunnel junctions depends on the spin polarization of the ferromagnetic electrodes used. The MR increases with increasing spin polarization of the electrode materials involved. This resulted in revived research interest 
in the class of half-metallic ferromagnets such as Heusler alloys [3], manganites [4], and $\mathrm{CrO}_{2}$ [5-10]. In this paper we present thin-film growth investigations as well as a magnetic and magnetotransport characterization of $\mathrm{CrO}_{2}$ films on $\mathrm{Al}_{2} \mathrm{O}_{3}(0001)$ and $\mathrm{TiO}_{2}(100)$ substrates .

The $\mathrm{CrO}_{2}$ films show three characteristic MR regimes [11]. At low magnetic fields $(<0.1 \mathrm{~T})$ and at low temperatures, intergrain tunnelling magnetoresistance (ITMR) dominates, whereas in the high-field regime ordinary Lorentz MR is the dominant contribution to the MR, which can be related to the crystalline properties (i.e. mean free path) of the films. In the intermediate-field range spin-dependent scattering at loose spins in the vicinity of grain boundaries seems to be the leading source of negative MR contribution and increases with increasing temperature. The observed linear and negative MR at high temperatures can be interpreted in terms of a recently proposed double-exchange mechanism of $\mathrm{CrO}_{2}[11,12,16]$. The magnitude of the anisotropic MR (AMR) due to spin-orbit interaction is less than $0.1 \%$ over the whole temperature range investigated.

\section{General properties}

$\mathrm{CrO}_{2}$ crystallizes in the tetragonal rutile-type structure (space group: $P 4_{2} / \mathrm{mnm}$ ) where the chromium atoms form a tetragonal unit cell. The $\mathrm{Cr}$ sites are octahedrally surrounded by oxygen atoms. The lattice parameters of the unit cell are $a=4.421 \AA$ and $c=2.916 \AA$ [13]. The $\mathrm{CrO}_{2}$ phase is a metastable oxide phase that degrades at temperatures above $400 \mathrm{~K}$ and atmospheric pressure into the thermodynamically more stable $\mathrm{Cr}_{2} \mathrm{O}_{3}$ phase $(a=4.951 \AA$; $c=13.566 \AA$; space group: $R \overline{3} c$ ) [14]. Therefore the preparation process is very sensitive to changes in temperature and oxygen pressure [15].

$\mathrm{Cr}_{2} \mathrm{O}_{3}$ is an antiferromagnetic $\left(T_{N}=307 \mathrm{~K}\right)$ and insulating compound, whereas $\mathrm{CrO}_{2}$ shows ferromagnetism $\left(T_{C}=393 \mathrm{~K}\right)$ [5] and metal-like electronic behaviour. The magnetic saturation moment of $\mathrm{CrO}_{2}$ is approximately $2 \mu_{B}$ per $\mathrm{Cr}^{4+}$ ion [15]. The ferromagnetic ordering has recently been interpreted in terms of a double-exchange mechanism $[11,12,16]$. For the electronic configuration one assumes one localized electron in a $x y$-orbital and one itinerant electron $\left((y z+z x)\right.$-orbital). The delocalized d electron of the $\mathrm{Cr}^{4+}$ ion is $\pi$-bonded with two $\mathrm{O}$ 2 p orbitals. This forms a partially filled d-p $\pi$-band at the Fermi energy $E_{F}$. The ferromagnetic coupling between the localized spins is mediated by the spin of the itinerant electrons. A recent $a b$ initio calculation based on local spin-density approximation which includes explicitly an on-site correlation term (LSDA $+U$ ) predicts the energy gap in the minority-spin band to be $2.8 \mathrm{eV}$ [12]. Ultraviolet photoemission (UPS) and bremsstrahlung isochromat spectra (BIS) are in good agreement with the LSDA $+U$ calculation [17]. Nearly $100 \%$ spin polarization has been found $2 \mathrm{eV}$ below $E_{F}$ in spin-polarized photoemission experiments [18], whereas approximately $90 \%$ has been observed near $E_{F}$ using a superconducting point-contact method [4].

The temperature-dependent resistivity of $\mathrm{CrO}_{2}$ varies with $\rho_{0}+\alpha T^{2}+\beta T^{\gamma}$ as expected for a correlated fully spin-polarized $\mathrm{d}$ band [19]. The $T^{2}$-term can be expected for scattering of highly correlated electrons and is the leading temperature dependence between approximately 150 and $330 \mathrm{~K}$ [19-22]. At higher temperatures, electron-magnon scattering (a two-magnon process) contributes with a $T^{3.5}$-dependence $[19,23]$.

The low-temperature Hall effect $(T \leqslant 50 \mathrm{~K})$ exhibits at magnetic fields larger than $1 \mathrm{~T}$ a sign reversal from positive to negative [24]. This is interpreted within a two-band model with the presence of highly mobile holes and less mobile electrons [24]. In comparison with that of manganites, the $\mathrm{MR}$ of $\mathrm{CrO}_{2}$ at higher fields $(>1 \mathrm{~T})$ is significantly smaller $(<-5 \%$ at $4.2 \mathrm{~K}$ ) [20] because of the lack of a Jahn-Teller effect. Films [22, 25] and polycrystalline $\mathrm{CrO}_{2}$ powder samples [26-29] exhibit a large ITMR of up to $-50 \%$ at $5 \mathrm{~K}$, which has been discussed in terms of the half-metallic spin-polarized electronic structure of $\mathrm{CrO}_{2}$. 


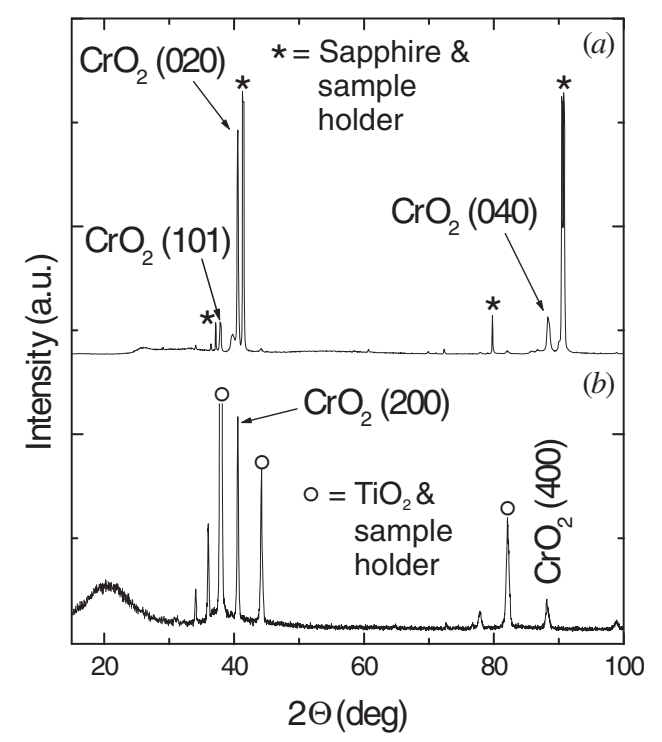

Figure 1. (a) An x-ray diffraction pattern of a $\mathrm{CrO}_{2}$ film deposited on an $\mathrm{Al}_{2} \mathrm{O}_{3}(0001)$ substrate. The dominant (020) and (040) peaks indicate an $a$-axis texture of the $\mathrm{CrO}_{2}$ film. (b) An x-ray diffraction pattern of an $a$-axis-oriented $\mathrm{CrO}_{2}$ film deposited on a $\mathrm{TiO}_{2}(100)$ substrate.

\section{Film growth}

The $\mathrm{CrO}_{2}$ films have been prepared by a chemical vapour deposition (CVD) technique proposed by Ishibashi et al [30]. As substrates, either $\mathrm{Al}_{2} \mathrm{O}_{3}(0001)(a=4.754 \AA$; $c=12.990 \AA$; space group: $R \overline{3} c)$ [31] or isostructural $\mathrm{TiO}_{2}(100)(a=4.592 \AA ; c=2.959 \AA$; space group: $\mathrm{P}_{2} / \mathrm{mnm}$ ) [32] have been used. These substrates were annealed at $1000^{\circ} \mathrm{C}$ for several hours before starting the deposition process. During deposition the substrate is oriented at an angle of $30^{\circ}$ with respect to the horizontal axis of the tube furnace. The reaction vessel used consists of a quartz glass tube with a length of $1 \mathrm{~m}$ and an inner diameter of $30 \mathrm{~mm}$. The temperature was controlled by $\mathrm{NiCr} \mathrm{Ni}$-thermocouples. $\mathrm{CrO}_{3}$ is decomposed at a temperature of $260{ }^{\circ} \mathrm{C}$ within a two-zone tube furnace. A well controlled oxygen flow $\left(0.51 \mathrm{~min}^{-1}\right)$ transports the decomposed precursor material $\left(\mathrm{CrO}_{3}\right)$ and its intermediate oxide phases $\left(\mathrm{Cr}_{2} \mathrm{O}_{5}, \mathrm{Cr}_{3} \mathrm{O}_{8}\right)$ into the deposition zone where the substrate is placed. The substrate temperature is adjusted to $390{ }^{\circ} \mathrm{C}$ enabling the growth of $\mathrm{CrO}_{2}$. The growth of a $200 \mathrm{~nm}$ thick $\mathrm{CrO}_{2}$ film applying this CVD process takes several hours.

\subsection{Structural characterization of (010)-oriented $\mathrm{CrO}_{2}$ films on $\mathrm{Al}_{2} \mathrm{O}_{3}(0001)$ substrates}

The crystallographic properties and the phase purity of the $\mathrm{CrO}_{2}$ films were characterized by x-ray diffractometry (XRD) using a Seifert Iso-Debyeflex 2002 diffractometer. A typical x-ray diffraction pattern (figure 1(a)) shows only dominant $\mathrm{CrO}_{2}(020)$ and $\mathrm{CrO}_{2}(040)$ peaks indicating a preferred $a$-axis growth. Peaks labelled with a star $(\star)$ are due to the underlying $\mathrm{Al}_{2} \mathrm{O}_{3}(0001)$ substrate and the sample holder. Peaks of smaller intensity are due to small deviations from the $a$-axis texture of $\mathrm{CrO}_{2}$.

For the transmission electron microscopy (TEM) analysis, a Philips CM20 Twin transmission electron microscope $(200 \mathrm{kV}$, point resolution $0.27 \mathrm{~nm})$ was used. The crosssection specimen preparation was performed in the following sequence: protecting the film 


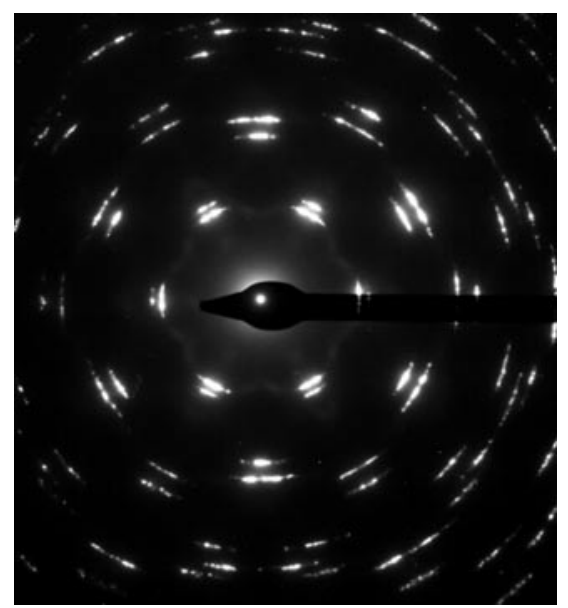

Figure 2. A plan-view electron diffraction pattern of a $\mathrm{CrO}_{2}$ film on an $\mathrm{Al}_{2} \mathrm{O}_{3}(0001)$ substrate with the incident electron beam parallel to the [00.1] axis of the $\mathrm{Al}_{2} \mathrm{O}_{3}(0001)$ substrate showing a sixfold in-plane symmetry.

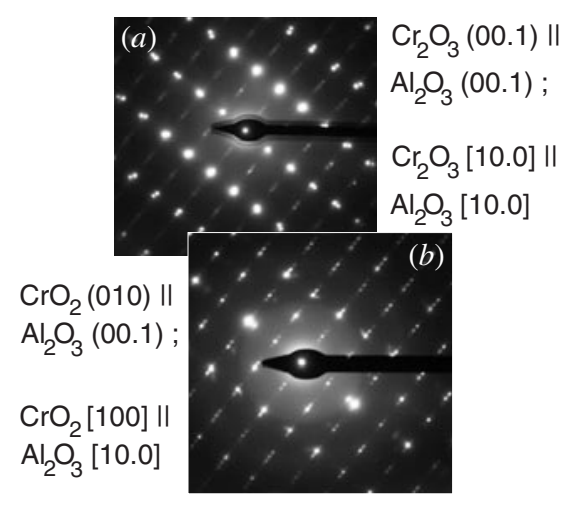

Figure 3. An electron diffraction pattern of a (010)-oriented $\mathrm{CrO}_{2}$ film on an $\mathrm{Al}_{2} \mathrm{O}_{3}(0001)$ substrate with the incident electron beam parallel to the in-plane [21.0] direction indicating (a) an initial $\mathrm{Cr}_{2} \mathrm{O}_{3}(0001)$ layer on the $\mathrm{Al}_{2} \mathrm{O}_{3}(0001)$ substrate with (b) a (010) orientation of the subsequent $\mathrm{CrO}_{2}$ layer.

surface by gluing a piece of silicon wafer on top, sawing off pieces $500 \mu \mathrm{m}$ wide and $2 \mathrm{~mm}$ long, grinding down to a thickness of less than $50 \mu \mathrm{m}$, mechanical polishing, gluing to a supporting ring, mechanical polishing of the second side, and finally thinning to electron transparency by means of an argon beam (energy $4 \mathrm{keV}$, reduced to $2 \mathrm{keV}$ for final thinning). The electron diffraction pattern of $\mathrm{CrO}_{2}$ films with the incoming electron beam parallel to the [00.1] axis of the substrate shows a sixfold in-plane symmetry (figure 2). For a (010)-oriented $\mathrm{CrO}_{2}$ layer an observed sixfold in-plane symmetry has to be related to a formation of twin boundaries due to the in-plane symmetry of the $\mathrm{Al}_{2} \mathrm{O}_{3}(0001)$ substrate or an underlying $\mathrm{Cr}_{2} \mathrm{O}_{3}(0001)$ layer. For further clarification, electron diffraction measurements have been performed with the incoming beam parallel to the surface of the film. As shown in figure 3(a), a $c$-axis-oriented $\mathrm{Cr}_{2} \mathrm{O}_{3}$ layer has been identified close to the $\mathrm{Al}_{2} \mathrm{O}_{3}(0001)$ substrate. This $\mathrm{Cr}_{2} \mathrm{O}_{3}$ layer grows isostructurally to the $\mathrm{Al}_{2} \mathrm{O}_{3}$ substrate $\left(\mathrm{Cr}_{2} \mathrm{O}_{3}(0001) \| \mathrm{Al}_{2} \mathrm{O}_{3}(0001)\right)$.

Electron diffraction analysis with the incoming beam parallel to $\mathrm{Cr}_{2} \mathrm{O}_{3}$ [21.0] (see 


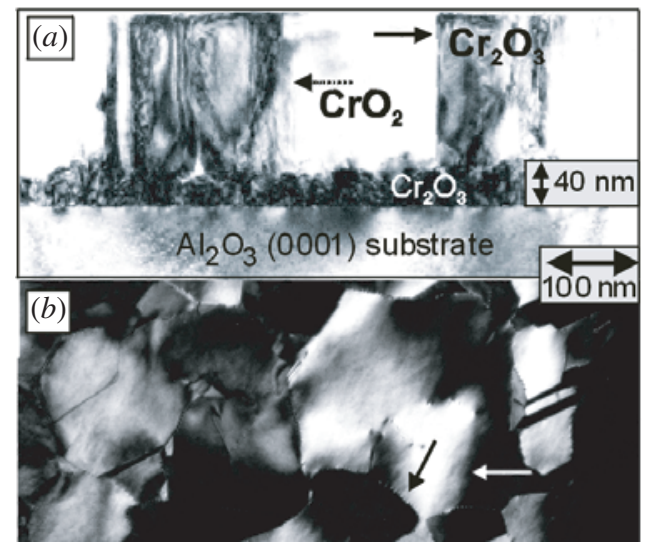

Figure 4. (a) A TEM (cross-section) image of a $\mathrm{CrO}_{2}$ film on $\mathrm{Al}_{2} \mathrm{O}_{3}(0001)$. A thin $\mathrm{Cr}_{2} \mathrm{O}_{3}$ layer at the film/substrate interface is visible before the onset of a columnar-like growth of $\mathrm{CrO}_{2}$. The strong contrast between two crystallites is given by the contact of $\mathrm{CrO}_{2}$ and $\mathrm{Cr}_{2} \mathrm{O}_{3}$ crystallites (black arrow). The gradual contrast change (dotted black arrow) is due to grain boundaries of differently oriented $\mathrm{CrO}_{2}$ crystallites. (b) A TEM (top view) image of a $\mathrm{CrO}_{2}$ film on $\mathrm{Al}_{2} \mathrm{O}_{3}(0001)$ ). The white arrow indicates grain boundaries between $\mathrm{CrO}_{2}$ crystallites with different in-plane orientation. The black arrow indicates a sharp interface between $\mathrm{CrO}_{2}$ and $\mathrm{Cr}_{2} \mathrm{O}_{3}$ crystallites.

figure 3(b)) shows that on top of the $\mathrm{Cr}_{2} \mathrm{O}_{3}(0001)$ initial layer one $a$-axis of $\mathrm{CrO}_{2}$ is oriented parallel to the $a$-axis of $\mathrm{Cr}_{2} \mathrm{O}_{3}\left(\mathrm{CrO}_{2}(010) \| \mathrm{Cr}_{2} \mathrm{O}_{3}(0001) ; \mathrm{CrO}_{2}\right.$ [100] $\| \mathrm{Cr}_{2} \mathrm{O}_{3}$ [10.0]). The [100] orientation of $\mathrm{CrO}_{2}$ crystallites along the three equivalent in-plane $a$-axes of $\mathrm{Cr}_{2} \mathrm{O}_{3}(0001)$ results in a crystalline twin formation and the apparent sixfold symmetry of the $\mathrm{CrO}_{2}$ layer as demonstrated in figure 2. The peak width in figure 2 indicates a small in-plane misalignment of the $\mathrm{CrO}_{2}$ unit cell.

The symmetry determined for the two different chromium oxide layers leads to the following interpretation of TEM images. Figure 4 shows (a) a cross-section and (b) a planview image of a $200 \mathrm{~nm}$ thick $\mathrm{CrO}_{2}$ film on $\mathrm{Al}_{2} \mathrm{O}_{3}(0001)$. The TEM cross-section shows an initial growth of an epitaxial $\mathrm{Cr}_{2} \mathrm{O}_{3}(0001)$ layer less than $40 \mathrm{~nm}$ thick directly on the substrate. After this initial $\mathrm{Cr}_{2} \mathrm{O}_{3}(0001)$ layer growth, a columnar-like growth of $\mathrm{CrO}_{2}$ grains is found (see figure 4(a)). The columnar growth of the $\mathrm{CrO}_{2}$ crystallites is intersected by some $\mathrm{Cr}_{2} \mathrm{O}_{3}$ inclusions. The gradual change in contrast within the $\mathrm{CrO}_{2}$ layer indicated by the dotted black arrow appears due to grain boundaries of differently in-plane-oriented $\mathrm{CrO}_{2}$ crystallites. Interphase boundaries between $\mathrm{CrO}_{2}$ and $\mathrm{Cr}_{2} \mathrm{O}_{3}$ lead to a sharp contrast as shown by the black arrow. In figure 4(b) the gradual contrast change indicates grain boundaries between $\mathrm{CrO}_{2}$ crystallites of different in-plane orientation (white arrow). In contrast, the ripple-like sharp interfaces (black arrow) are due to interphase boundaries between $\mathrm{CrO}_{2}$ and $\mathrm{Cr}_{2} \mathrm{O}_{3}$ crystallites.

Presumably the initial $\mathrm{Cr}_{2} \mathrm{O}_{3}(0001)$ layer is deposited under the unstable temperature conditions that prevail while heating the precursor $\mathrm{CrO}_{3}$. The growth of this initial $\mathrm{Cr}_{2} \mathrm{O}_{3}$ layer is apparently also favoured by the small lattice mismatch of $\left(a_{\mathrm{Cr}_{2} \mathrm{O}_{3}}-a_{\mathrm{Al}_{2} \mathrm{O}_{3}}\right) / a_{\mathrm{Al}_{2} \mathrm{O}_{3}}=4 \%$ between the $\mathrm{Cr}_{2} \mathrm{O}_{3} a$-axis and the $a$-axis of the underlying $\mathrm{Al}_{2} \mathrm{O}_{3}$ substrate. For further clarification of the in-plane twin boundary formation of (010)-oriented $\mathrm{CrO}_{2}$ films, a scanning electron microscopy (SEM) image of large $\mathrm{CrO}_{2}$ crystallites is shown in figure 5(a). The $c$-axes of different grains are oriented along the three equivalent in-plane axes of $\mathrm{Cr}_{2} \mathrm{O}_{3}(0001)$. The preferred growth direction of $\mathrm{CrO}_{2}$ crystallites is the crystallographic $c$-axis, which leads to a needle-like morphology of the $\mathrm{CrO}_{2}$ crystallites. This is similar to the case for $\mathrm{CrO}_{2}$ powder particles which are also preferentially extended along the $c$-axis direction. A schematic model 

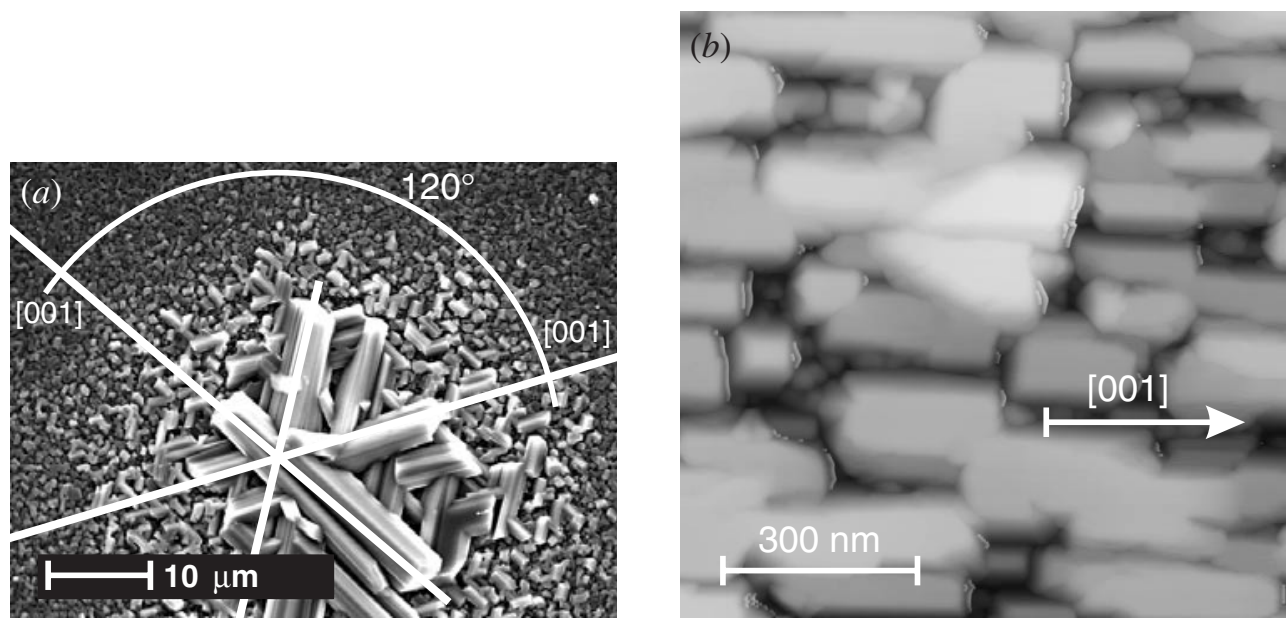

Figure 5. (a) A scanning electron microscopy image of (010)-oriented $\mathrm{CrO}_{2}$ crystallites on a $\mathrm{Cr}_{2} \mathrm{O}_{3}(0001)$ initial layer grown on $\mathrm{Al}_{2} \mathrm{O}_{3}$. (b) An $\mathrm{AFM}$ image of (100)-oriented $\mathrm{CrO}_{2}$ crystallites grown on isostructural $\mathrm{TiO}_{2}(100)$ substrates.

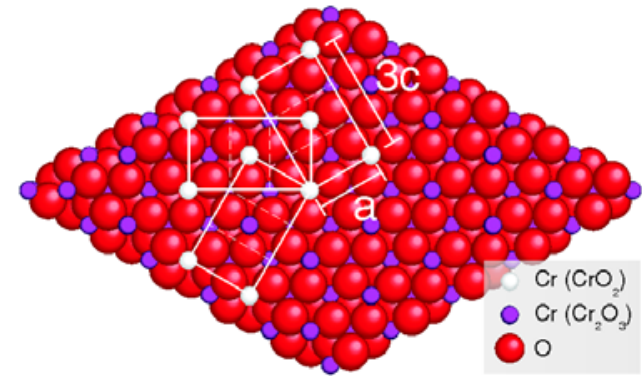

Figure 6. The schematic model of $\mathrm{CrO}_{2}(010)$ growth on a $\mathrm{Cr}_{2} \mathrm{O}_{3}(0001)$ initial layer deposited on $\mathrm{Al}_{2} \mathrm{O}_{3}(0001)$.

(This figure is in colour only in the electronic version)

of the observed $\mathrm{Cr}_{2} \mathrm{O}_{3}(0001)$ and $\mathrm{CrO}_{2}(010)$ growth on an $\mathrm{Al}_{2} \mathrm{O}_{3}(0001)$ substrate is shown in figure 6. First a $\mathrm{Cr}_{2} \mathrm{O}_{3}(0001)$ layer continues the in-plane symmetry of the sapphire substrate. This initial $\mathrm{Cr}_{2} \mathrm{O}_{3}(0001)$ layer initiates three nearly equivalent in-plane orientations for the $c$-axis direction of the $\mathrm{CrO}_{2}$ unit cell.

\subsection{Structural characterization of (100)-oriented $\mathrm{CrO}_{2}$ films on $\mathrm{TiO}_{2}(100)$ substrates}

In contrast to the growth of $\mathrm{CrO}_{2}$ on $\mathrm{Al}_{2} \mathrm{O}_{3}(0001)$ substrates, the growth of $\mathrm{CrO}_{2}$ on isostructural $\mathrm{TiO}_{2}(100)$ leads to epitaxial $\mathrm{CrO}_{2}(100)$ films. The lattice mismatches between the $a$ - and $c$-axes for the $\mathrm{CrO}_{2}$ film and the substrate are only -3.79 and $-1.48 \%$, respectively [5]. $\mathrm{X}$-ray diffraction shows the $a$-axis orientation of the $\mathrm{CrO}_{2}$ films (see figure 1(b)). The atomic force microscopy (AFM) image in figure 5(b) reveals extended $\mathrm{CrO}_{2}$ crystallites with an overall twofold symmetry, as expected for the growth on isostructural $\mathrm{TiO}_{2}(100)$ substrates. The in-plane $c$-axis of the $\mathrm{CrO}_{2}$ crystallites is indicated in figure 5(b) with an arrow.

Figure 7 shows a TEM image (cross-section) of an epitaxial $100 \mathrm{~nm}$ thick $\mathrm{CrO}_{2}(100)$ film grown on an isostructural $\mathrm{TiO}_{2}(100)$ substrate. In contrast to the growth of $\mathrm{CrO}_{2}$ on 


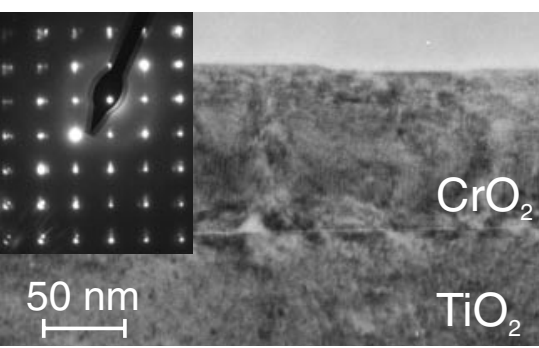

Figure 7. A TEM (cross-section) image of a $\mathrm{CrO}_{2}(100)$ film on a $\mathrm{TiO}_{2}(100)$ substrate. The inset shows an electron diffraction pattern with the incident electron beam parallel to the [001] direction of the substrate.

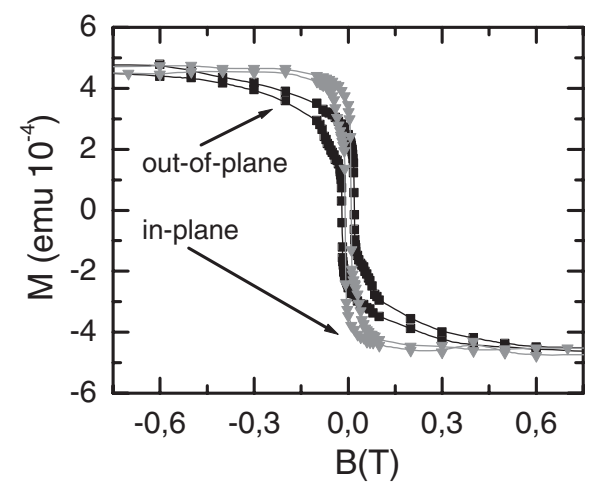

Figure 8. SQUID magnetometry on an array of $20 \mu \mathrm{m}$ wide, $200 \mathrm{~nm}$ thick $\mathrm{CrO}_{2}$ wires at $T=5 \mathrm{~K}$ clearly shows an in-plane magnetic easy axis.

$\mathrm{Al}_{2} \mathrm{O}_{3}(0001)$ substrates, no $\mathrm{Cr}_{2} \mathrm{O}_{3}$ initial layer at the substrate interface and no $\mathrm{Cr}_{2} \mathrm{O}_{3}$ inclusions within the $\mathrm{CrO}_{2}$ layer have been observed. The inset of figure 7 exhibits an electron diffraction pattern with an incident electron beam along the [001] direction of the $\mathrm{TiO}_{2}$ substrate imaging the fourfold in-plane symmetry of the $\mathrm{CrO}_{2}(001)$ and $\mathrm{TiO}_{2}(001)$ plane.

\section{Magnetic and magnetotransport properties}

For investigating the magnetotransport properties of $200 \mathrm{~nm}$ thick $\mathrm{CrO}_{2}$ films, well defined four-probe transport structures have been microfabricated using electron-beam lithography techniques (Philips XL30, Elphy Plus FE Raith) in conjunction with an ion milling process $\left(\sim 500 \mathrm{eV} \mathrm{Ar}^{+}\right.$ions). For all transport measurements the wire width and the distance between the voltage probes are 20 and $120 \mu \mathrm{m}$, respectively. For studying the magnetization reversal of the $\mathrm{CrO}_{2}$ wires a $5 \times 5 \mathrm{~mm}^{2}$ array of $20 \mu \mathrm{m}$ wide wires (periodicity: $50 \mu \mathrm{m}$ ) has been fabricated.

The magnetization reversal of an array of $20 \mu \mathrm{m}$ wide $\mathrm{CrO}_{2}$ wires grown on $\mathrm{Al}_{2} \mathrm{O}_{3}(0001)$ was investigated by SQUID magnetometry (Quantum Design MPMS XL). Both in-plane and out-of-plane magnetization have been measured as functions of an applied magnetic field (see figure 8). Over the whole temperature range $(4.2-390 \mathrm{~K})$ the wires show a magnetic hysteresis with an in-plane magnetic easy axis. The ferromagnetic ordering temperature is $T_{C}=385 \mathrm{~K}$ which is slightly reduced compared to the bulk value of $391 \mathrm{~K}$ [15].

The MR measurements were performed in a variable-temperature high-field cryostat 

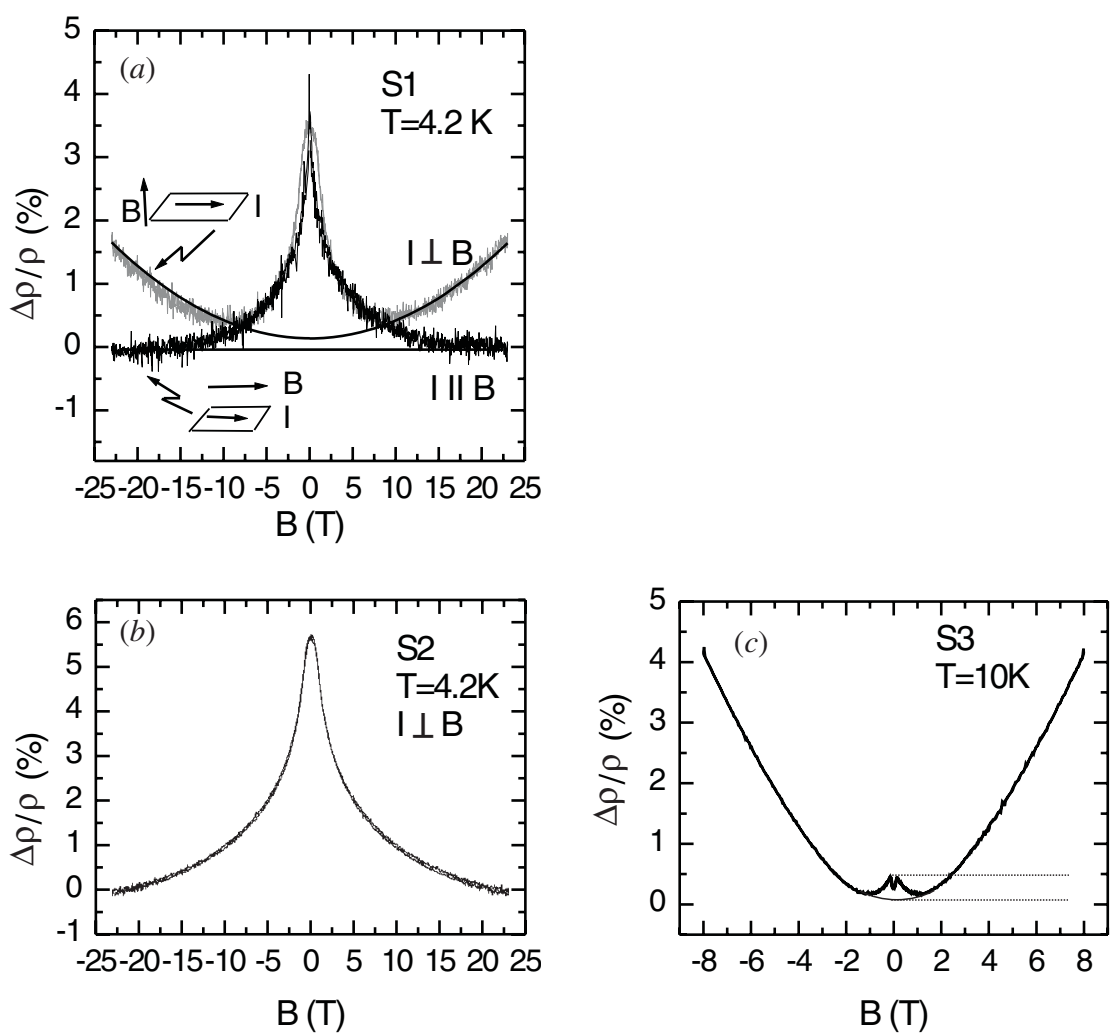

Figure 9. (a) The high field $\mathrm{MR}$ of a $20 \mu \mathrm{m}$ wide $\mathrm{CrO}_{2}$ wire $\left(\mathrm{S} 1\right.$; grown on $\left.\mathrm{Al}_{2} \mathrm{O}_{3}\right)$ at $4.2 \mathrm{~K}$ in the longitudinal and perpendicular field geometry, (b) the high field $\mathrm{MR}$ of a $20 \mu \mathrm{m}$ wide $\mathrm{CrO}_{2}$ wire ( $\mathrm{S} 2$; grown on $\mathrm{Al}_{2} \mathrm{O}_{3}$ ) of a lesser crystalline quality at $4.2 \mathrm{~K}$ in the perpendicular field geometry, and (c) the high field MR of sample $\left(\mathrm{S} 3\right.$; grown on $\left.\mathrm{TiO}_{2}\right)$ at $10 \mathrm{~K}$ in the perpendicular field geometry.

$( \pm 10 \mathrm{~T})$ with in situ sample rotation capabilities. The measurements up to $\pm 23 \mathrm{~T}$ were performed at the Grenoble High Magnetic Field Laboratory. The applied field was oriented outof-plane (perpendicular), in-plane parallel to the long axis of the wire (longitudinal transport current direction), and in-plane perpendicular to the long axis (transverse). Four-probe ac and dc measurements with bias currents $\boldsymbol{J}$ of $100 \mu \mathrm{A}$ were employed and the magnetic history of the samples was carefully controlled.

Figure 9 shows representative high-field MR results on $20 \mu \mathrm{m}$ linewidth wires at $4.2 \mathrm{~K}$ in the perpendicular and longitudinal field geometries, respectively. Sample S1 is a sample with better crystalline properties than sample $\mathrm{S} 2$, both grown on $\mathrm{Al}_{2} \mathrm{O}_{3}(0001)$, which can be seen from the residual resistivities at $4.2 \mathrm{~K}\left(\rho_{\mathrm{S} 1,4.2 \mathrm{~K}}=223 \mu \Omega \mathrm{cm}, \rho_{\mathrm{S} 2,4.2 \mathrm{~K}}=544 \mu \Omega \mathrm{cm}\right)$ and the residual resistivity ratios $\left(\mathrm{RRR}=R_{300 \mathrm{~K}} / R_{4.2 \mathrm{~K}}\right)$ of $\mathrm{RRR}_{\mathrm{S} 1}=5.9$ and $\mathrm{RRR}_{\mathrm{S} 2}=3.2$, respectively.

Figure 9(a) compares the high-field MR of sample S1 at $4.2 \mathrm{~K}$ in the perpendicular and longitudinal field geometries. Below $\pm 10 \mathrm{~T}$ in both field geometries a similar steep decrease of the resistivity with increasing magnetic field appears. The magnitude of the observed negative $\operatorname{MR}([\rho(10 \mathrm{~T})-\rho(0 \mathrm{~T})] / \rho(10 \mathrm{~T})=\Delta \rho / \rho)$ is in both orientations approximately $\Delta \rho / \rho=-3.5 \%$. The negative MR saturates in longitudinal fields larger than approximately $\pm 10 \mathrm{~T}$. Above $\pm 10 \mathrm{~T}$ in the perpendicular field geometry $(\boldsymbol{I} \perp \boldsymbol{B})$ the quadratic increase of the 
resistivity is due to the ordinary Lorentz MR (see figure 9(a)), which is in general anisotropic (i.e. dependent on the relative orientation of $\boldsymbol{I}$ and $\boldsymbol{B}$ ) [33,34]. In the longitudinal field geometry $(\boldsymbol{I} \| \boldsymbol{B})$ no significant Lorentz MR can be resolved. In contrast, sample $\mathrm{S} 2$ shows no significant Lorentz MR contribution at $4.2 \mathrm{~K}$ in the perpendicular field geometry $(\boldsymbol{I} \perp \boldsymbol{B})$ (see figure 9 (b)). Over the whole field range the resistivity decreases and even at $\pm 23 \mathrm{~T}$ no saturation of this negative MR contribution has been achieved. For sample S2 the negative MR $[\rho(23 \mathrm{~T})-$ $\rho(0 \mathrm{~T})] / \rho(23 \mathrm{~T})$ is larger than for sample $\mathrm{S} 1$ and has been determined as $\Delta \rho / \rho=-5.4 \%$.

In magnetic thin films the Lorentz MR can directly be related to the crystalline quality of the films $[33,34]$. The field-dependent resistivity with the magnetic field perpendicular (parallel) to the current can be described in a first approximation by

$\rho_{\perp, \|}(B, T)=\rho_{\perp, \|}(0, T)\left[1+F_{\perp, \|}\left(\frac{B}{\rho_{\perp, \|}(0, T)}\right)\right] \cong \rho_{\perp, \|}(0, T)\left[1+\zeta\left(\frac{B}{\rho_{\perp, \|}}\right)^{2}\right]$.

Here $B$ is the internal field in the ferromagnet, $\rho_{\perp, \|}(0, T)$ the resistivity at zero internal field, and $F_{\perp, \|}$ is known as the Kohler function, which parametrizes the Lorentz MR in terms of $B / \rho \propto \omega \tau$, the cyclotron frequency times the relaxation time [35], and $\zeta$ is a fitting parameter. From this relationship one directly understands that the lower resistivity (longer mean free path) of sample S1 leads to a larger Lorentz MR at a given temperature and is probably due to a smaller content of grain boundaries (loose spins near grain boundaries and defects) and paramagnetic impurities.

A clear indication of the better crystalline quality of the $\mathrm{CrO}_{2}$ films grown on $\mathrm{TiO}_{2}$ (sample S3) is the large RRR - of 18-compared with that for $\mathrm{CrO}_{2}$ films on $\mathrm{Al}_{2} \mathrm{O}_{3}(0001)$ ( $\mathrm{RRR}$ of 3 to 6). In comparison with that for the $\mathrm{CrO}_{2}$ film on $\mathrm{Al}_{2} \mathrm{O}_{3}$, the magnitude of the Lorentz MR contribution is much larger and has already been observed in the perpendicular field geometry above $\pm 1 \mathrm{~T}$ at $10 \mathrm{~K}$ (figure $9(\mathrm{c})$ ). The negative MR contribution is already saturated above $\pm 1 \mathrm{~T}$ and is, at only $\Delta \rho / \rho=-0.5 \%$, approximately one order of magnitude smaller than for the films grown on $\mathrm{Al}_{2} \mathrm{O}_{3}$. This negative $\mathrm{MR}$ contribution at low temperatures obviously correlates with the crystalline quality (grain boundary density) of the samples.

From figure 9(a) one can estimate that the $\operatorname{AMR}\left[\rho_{\|}(B=0, T)-\rho_{\perp}(B=0, T)\right] / \rho(0, T)$ gives no significant contribution to the total $\mathrm{MR}$ of $\mathrm{CrO}_{2}$. The AMR has its origin in spinorbit coupling, depends on the relative orientation of $\boldsymbol{I}$ and $\boldsymbol{M}$, and can be determined by extrapolating the field-dependent resistivity back to zero internal field [36]. A quadratic fit of the high-field data in the perpendicular geometry above $\pm 10 \mathrm{~T}$ produced for this purpose is shown in figure 9(a); for the longitudinal case this is a field-independent horizontal line (due to the lack of a Lorentz MR contribution). At zero applied field the two extrapolated resistivities are nearly equal $\left(\rho_{\|}(B=0, T) \cong \rho_{\perp}(B=0, T)\right)$, indicating a negligible AMR contribution to the total MR in $\mathrm{CrO}_{2}$. This observation has been made over a wide temperature range $(4.2-100 \mathrm{~K})$.

As is known from the analysis of the MR in highly spin-polarized half-metallic manganites, intergrain tunnelling MR exhibits low- and high-field regimes [37,38]. This phenomenon is characterized by an initial drop of the resistivity with applied magnetic field and a subsequent slower decrease at higher fields. In the low-field regime the MR is hysteretic and shows its maximum at the coercive field. The MR can be qualitatively understood within Jullière's model [2] for ferromagnetic/insulator/ferromagnetic tunnel junctions considering only direct (elastic) tunnelling processes. Figure 10 shows the low-field ITMR of sample $\mathrm{S} 2$ at $150 \mathrm{~K}$ in the longitudinal field geometry. The low-field ITMR is given by $\left[\rho_{0}-\rho\left(H_{C}\right)\right] / \rho\left(H_{C}\right)=\Delta \rho / \rho$, where $\rho_{0}$ is the extrapolated resistivity at $B=0$ (see figure 10). A systematic determination of the temperature dependence of $\Delta \rho / \rho$ in sample $\mathrm{S} 1$ and $\mathrm{S} 2$ is plotted in figure 11 (left-hand axis). At $4.2 \mathrm{~K}$ the low-field ITMR is approximately $-0.5 \%$ and rapidly drops with increasing 


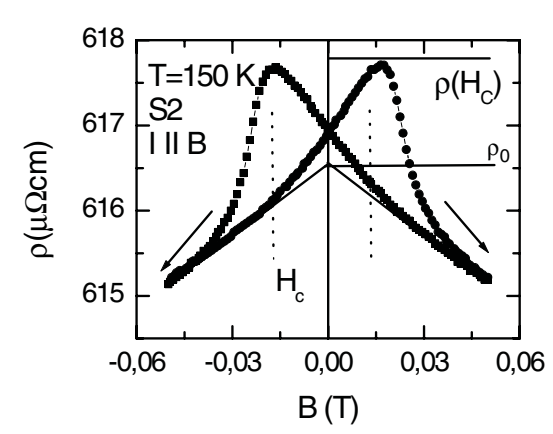

Figure 10. The hysteretic low-field MR due to intergrain tunnelling of a single $20 \mu \mathrm{m}$ wide $\mathrm{CrO}_{2}$ wire (S2; grown on $\mathrm{Al}_{2} \mathrm{O}_{3}$ ) in the longitudinal field configuration $(\boldsymbol{I} \| \boldsymbol{B})$ at $150 \mathrm{~K}$.

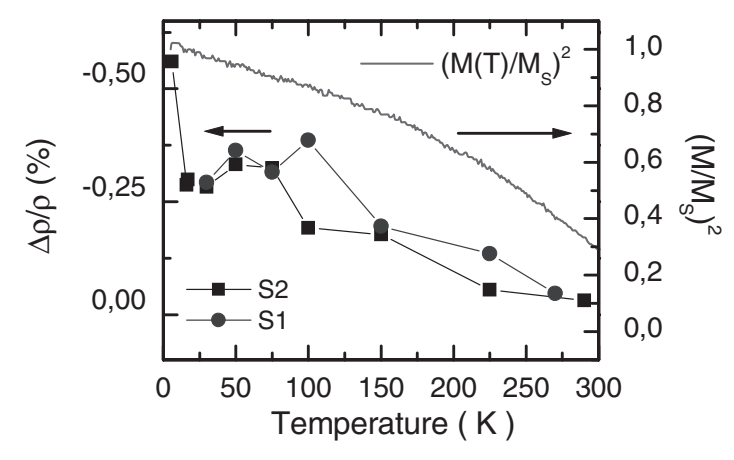

Figure 11. The temperature dependence of the low-field ITMR $\Delta \rho / \rho=\left(\rho_{0}-\rho\left(H_{C}\right)\right) / \rho\left(H_{C}\right)$ (left-hand axis) and $\left(M / M_{S}\right)^{2}$ (right-hand axis) of samples $\mathrm{S} 1$ and $\mathrm{S} 2$ (both grown on $\mathrm{Al}_{2} \mathrm{O}_{3}$ ).

temperature. The two samples show comparable magnitudes and temperature dependences of the ITMR contribution.

The analysis of the field dependence of the low-field MR $\left[\rho(H)-\rho\left(H_{C}\right)\right] / \rho\left(H_{C}\right)$ versus the corresponding projection of the local magnetization onto the $H$-axis $\left(M(H) / M_{S}\right)$ at $50 \mathrm{~K}$ in figure 12 emphasizes that the origin of the low-field ITMR is the misalignment of the relative orientations of the magnetic axes of individual $\mathrm{CrO}_{2}$ crystallites [25-27,39]. This is analogous to the situation in granular-like giant-magnetoresistance $(\mathrm{GMR})$ systems with $\left(M(H) / M_{S}\right)^{2}=$ $\langle\cos \Theta\rangle^{2}$, where $\Theta$ is the angle between the magnetization axis of a ferromagnetic particle and the external magnetic field, and $\langle\cos \Theta\rangle$ is the average value over the whole ensemble [40]. The solid curve in figure 12 is the fit $\Delta \rho / \rho=\left[\rho(H)-\rho\left(H_{C}\right)\right] / \rho\left(H_{C}\right)=-a\left(M(H) / M_{S}\right)^{2}$ with $a=-0.0045$. The $M(H) / M_{S}$ values were taken from a magnetic hysteresis loop for the whole wire array determined by SQUID magnetometry. The deviations for small $M(H) / M_{S}$ values are due to small differences between the averaged coercive fields of the whole wire array (determined by SQUID magnetometry) and that of the single transport wire. As also observed in $\mathrm{CrO}_{2}$ powder samples [26, 27], polycrystalline $\mathrm{CrO}_{2}$ films [25,39], and manganites $[37,38]$, the temperature dependence of the low-field ITMR does not follow $\left(M(T) / M_{S}\right)^{2}$ (see figure 11, right-hand axis), indicating that the temperature dependence of the surface magnetization near grain boundaries (reduced magnetic exchange) is presumably different from the bulk values. In contrast, the recently investigated ordered double-perovskite compound $\mathrm{Sr}_{2} \mathrm{FeMoO}_{6}$ shows, over the whole temperature range from 4.2 to $300 \mathrm{~K}$, an ITMR, which scales with $\left(M(T) / M_{S}\right)^{2}$, indicating that the magnetic coupling at grain boundaries is 


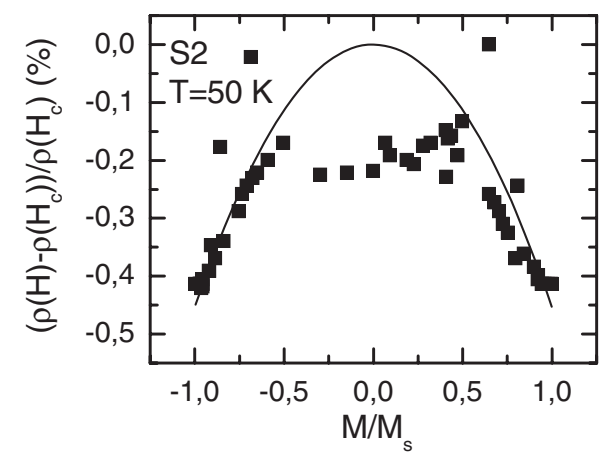

Figure 12. MR $\Delta \rho / \rho=\left[\rho(H)-\rho\left(H_{C}\right)\right] / \rho\left(H_{C}\right)$ of sample $\mathrm{S} 2$ (grown on $\mathrm{Al}_{2} \mathrm{O}_{3}$ ) versus net magnetization $M(H) / M_{S}$ at $T=50 \mathrm{~K}$. The solid curve is the fit $\Delta \rho / \rho=-a\left(M / M_{S}\right)^{2}$ with $a=-0.0045$.

similar to the bulk coupling $[41,42]$. At higher temperatures the number of direct tunnelling processes is strongly reduced by spin-flip scattering processes due to magnetic impurities and excitation of surface as well as bulk magnons [43] or hopping processes via localized states within the barrier [26], which results in the rapid drop of the low-field ITMR with increasing temperature. The antiferromagnetic nature of the grain boundaries $\left(\mathrm{Cr}_{2} \mathrm{O}_{3}\right.$ inclusions and oxygen deficiencies on the surface of $\mathrm{CrO}_{2}$ crystallites [44]) in $\mathrm{CrO}_{2}$ films (see section 3) supports this assumption.

At $300 \mathrm{~K}$ the MRs of $\mathrm{CrO}_{2}$ films on substrates of both types show linear decrease with increasing magnetic field (see figures 13(a) and 13(b)). In the case of the (010)-oriented $\mathrm{CrO}_{2}$ film on $\mathrm{Al}_{2} \mathrm{O}_{3}(0001)$ (see figure 13(a)) magnetic fields of $\pm 23 \mathrm{~T}$ are not sufficient for saturating the negative MR at $300 \mathrm{~K}$. The same observation has been made for the $\mathrm{CrO}_{2}$ film grown on $\mathrm{TiO}_{2}(100)$ (see figure 13(b)). The magnitude of the observed negative MR $[\rho(8 \mathrm{~T})-\rho(0 \mathrm{~T})] / \rho(8 \mathrm{~T})$ is $-3.7 \%$ (sample $\mathrm{S} 1$ ) and $-4.0 \%$ (sample $\mathrm{S} 2$ ) for the $\mathrm{CrO}_{2}$ films on $\mathrm{Al}_{2} \mathrm{O}_{3}(0001)$ and $-4.4 \%$ (sample S3) for the film on $\mathrm{TiO}_{2}(100)$. This negative MR contribution seems to be independent of the crystalline properties (grain boundary density) of the $\mathrm{CrO}_{2}$ films and supports the suggested double-exchange mechanism for $\mathrm{CrO}_{2}$ [12]. Therefore, paramagnetic impurities or weakly coupled spins near and within grain boundaries can be excluded from consideration as sources of this negative linear MR contribution. Also, the fact that $\pm 23 \mathrm{~T}$ is not sufficient to saturate this negative contribution at $300 \mathrm{~K}$ supports the assumption of an intrinsic source of the negative MR related to fundamental properties of $\mathrm{CrO}_{2}$.

The presence of this negative MR contribution over the whole temperature range and in all three field geometries is at variance with a previous interpretation, where a finite-size effect in the presence of a magnetic field has been employed to explain the decrease of resistivity in the intermediate-field range at low temperatures [20]. Such a negative MR is expected in ferromagnetic systems when the mean free path of the charge carriers is of the same order of magnitude as the structure size [45]. This mechanism should strongly depend on the field orientation and the sample geometry, which have not been observed for the microfabricated samples. For an estimate of the mean free path $l$, the following approximate relation [46] can be deduced from the Boltzmann transport theory:

$l=\frac{\sum\left|\vec{v}_{k}\right|^{2} \tau \delta\left(\epsilon_{k}\right)}{\sum\left|\vec{v}_{k}\right| \delta\left(\epsilon_{k}\right)} \cong \frac{(2 \pi)^{3} \hbar}{e^{2}} \frac{1}{A_{F S}^{\uparrow}+A_{F S}^{\downarrow}}\left(\frac{1}{\rho_{x x}}+\frac{1}{\rho_{y y}}+\frac{1}{\rho_{z z}}\right) \cong \frac{(2 \pi)^{3} \hbar}{e^{2}} \frac{1}{A_{F S}^{\uparrow}+A_{F S}^{\downarrow}} \frac{3}{\bar{\rho}}$ 

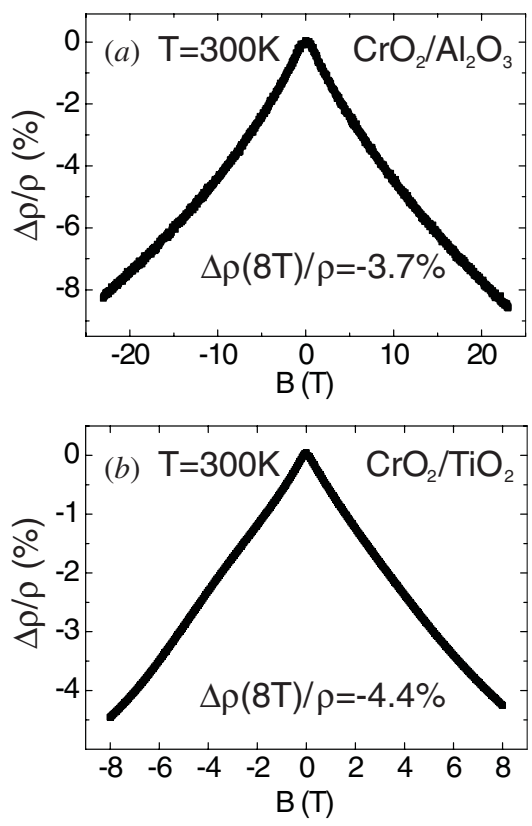

Figure 13. The linear high-field $\mathrm{MR}$ at $T=300 \mathrm{~K}$ of (a) a (010)-oriented $\mathrm{CrO}_{2}$ film grown on an $\mathrm{Al}_{2} \mathrm{O}_{3}(0001)$ substrate and (b) a (100)-oriented $\mathrm{CrO}_{2}$ film grown on a $\mathrm{TiO}_{2}(100)$ substrate.

where $A_{F S}^{\uparrow, \downarrow}, \tau, \vec{v}_{k_{F}}$, and $\bar{\rho}$ are the theoretical area of the Fermi surface, which can be calculated using density functional theory, the scattering time, the Fermi velocity, and the averaged resistivity, respectively. With a calculated Fermi surface area of $A_{F S}^{\uparrow, \downarrow} \cong 8.9 \AA^{-2}$ [46], the estimated mean free path (sample S1) at $4.2 \mathrm{~K}$ is only $7.8 \AA$ and decreases to $3.2 \AA$ at $300 \mathrm{~K}$; these are surprisingly short mean free paths. Nevertheless, for all temperatures the mean free path is significantly smaller than the sample size (especially at higher temperatures), so a finite-size effect cannot be the origin of the observed negative MR in the intermediate- and high-field ranges.

Such a linear decease of $\rho(H)$ at $300 \mathrm{~K}$ (see figure 13) has also been found for doped manganites and has recently been interpreted in terms of the double-exchange mechanism [47]. The MR of $\mathrm{CrO}_{2}$ at higher temperatures is orders of magnitude smaller than for the manganites due to the lack of a Jahn-Teller effect near $T_{C}$. In contrast to the case of the manganites, for $\mathrm{CrO}_{2}$ the positive slope of $\rho(T)$ does not significantly change near $T_{C}[7,22]$ - which would be an indication of electron localization due to a Jahn-Teller distortion. In order to explain the negative MR, the strong coupling between conduction electrons and localized core spins has to be taken into account [16]. An increasing magnetic field aligns adjacent spins which leads to an enhancement of the carrier mobility and, therefore, decreases the resistance. This MR due to the presence of a double-exchange mechanism decreases with decreasing temperature where the localized spins are more perfectly aligned and spin fluctuations are suppressed; this would explain the presence of the linear and negative MR contribution only at higher temperatures.

\section{Conclusions}

$\mathrm{CrO}_{2}$ thin films have been deposited on $\mathrm{Al}_{2} \mathrm{O}_{3}(0001)$ and $\mathrm{TiO}_{2}(100)$ substrates by CVD. Electron diffraction shows that on $\mathrm{Al}_{2} \mathrm{O}_{3}(0001) \mathrm{CrO}_{2}(010)$ grows epitaxially on an initial 
$\mathrm{Cr}_{2} \mathrm{O}_{3}(0001)$ layer, whereas the in-plane symmetry of $\mathrm{Cr}_{2} \mathrm{O}_{3}(0001)$ leads to a columnar growth mode with the presence of grain boundaries between $\mathrm{CrO}_{2}$ crystallites. The growth of $\mathrm{CrO}_{2}$ thin films on $\mathrm{TiO}_{2}(100)$ substrates leads to films with better crystalline quality. All present contributions to the MR have been determined between 4.2 and $300 \mathrm{~K}$ in magnetic fields up to $23 \mathrm{~T}$. The magnitude of the Lorentz MR strongly depends on the defect density of the sample, the AMR is less than $0.1 \%$, the intergrain tunnelling MR is small $(\leqslant-0.5 \%$ at $4.2 \mathrm{~K})$ in comparison with those for polycrystalline and powder samples, and spin-disorder scattering is the leading source of the linear and negative $\mathrm{MR}$ of the $\mathrm{CrO}_{2}$ films investigated. The linear and negative MR at higher temperatures is independent of the crystalline properties of the sample and consistent with the proposed double-exchange mechanism in $\mathrm{CrO}_{2}$ [12].

\section{Acknowledgments}

This work was supported by the German Federal Ministry for Education and Research 'BMBF' under grant No 13N7329. Parts of the work were carried out at the Grenoble High Magnetic Field Laboratory (Laboratoire des Champs Magnétiques Intenses du CNRS Grenoble).

\section{References}

[1] Schwarz K-H 1986 J. Phys. F: Met. Phys. 16 L211

[2] Jullière M 1975 Phys. Lett. A 54225

[3] de Groot R, Müller F, van Engen P and Buschow K H J 1983 Phys. Rev. Lett. 502024

[4] Soulen R J Jr et al 1998 Science 28285

[5] Li X W, Gupta A and Xiao G 1999 Appl. Phys. Lett. 75713

[6] Yang F Y et al 2000 Appl. Phys. Lett. 77286

[7] Li X W et al 1999 J. Appl. Phys. 855585

[8] DeSisto W J et al 2000 Appl. Phys. Lett. 763789

[9] Yang F Y et al 2001 Phys. Rev. B 63092403

[10] Spinu L et al 2000 Phys. Rev. B 628931

[11] Rüdiger U et al 2001 J. Appl. Phys. 897699

[12] Korotin M A, Anisimov V I, Khomskii D I and Sawatzky G A 1998 Phys. Rev. Lett. 804305

[13] Thamer B J, Douglass R M and Staritzky E 1957 J. Am. Chem. Soc. 79547

[14] Finger L W and Hazen R M 1980 J. Appl. Phys. 515362

[15] Chamberland B 1977 Crit. Rev. Solid State Sci. 7

[16] Zener C 1951 Phys. Rev. 82403

[17] Tsujioka T et al 1997 Phys. Rev. B 56 R15 509

[18] Kämper K P et al 1987 Phys. Rev. Lett. 592788

[19] Ranno L, Barry A and Coey J M D 1997 J. Appl. Phys. 815774

[20] Suzuki K and Tedrow P M 1998 Phys. Rev. B 5811597

[21] Suzuki K and Tedrow P M 1999 Appl. Phys. Lett. 74428

[22] Rabe M et al 2000 J. Magn. Magn. Mater. 211314

[23] Irkhin V Y and Katsnel'son M I 1994 Phys. Usp. 37659

[24] Watts S et al 2000 Phys. Rev. B 619621

[25] Hwang H and Cheong S-W 1997 Science 2781607

[26] Manoharan S S, Elefant D, Reiss G and Goodenough J B 1998 Appl. Phys. Lett. 72984

[27] Coey J M D et al 1998 Phys. Rev. Lett. 803815

[28] Dai J and Tang J 2001 Phys. Rev. B 63064410

[29] Dai J and Tang J 2001 Phys. Rev. B 63054434

[30] Ishibashi S, Namikawa T and Satou M 1979 Mater. Res. Bull. 1451

[31] Ishizawa N, Miyata T, Minato I, Marumo F and Iwai S 1980 Acta Crystallogr. B 36228

[32] Sabine T M and Howard C J 1982 Acta Crystallogr. B 38701

[33] Schwerer F and Silox J 1968 Phys. Rev. Lett. 20101

[34] Schwerer F and Silox J 1968 J. Appl. Phys. 392047

[35] Ziman J M 1960 Electrons and Phonons (Oxford: Clarendon) p 490 
[36] Campbell I and Fert A 1982 Ferromagnetic Materials vol 3, ed E Wohlfarth (Amsterdam: North-Holland)

[37] Lee S et al 1999 Phys. Rev. Lett. 824508

[38] Hwang H Y, Cheong S-W, Ong N P and Batlogg B 1996 Phys. Rev. Lett. 772041

[39] Gupta A, Li X W and Xiao G 2000 J. Appl. Phys. 876073

[40] Xiao J Q, Jiang J S and Chien C L 1992 Phys. Rev. Lett. 683749

[41] Kobayashi K-I et al 1998 Nature 395677

[42] Kim T H, Uehara M and Cheong S-W 1999 Appl. Phys. Lett. 741737

[43] Guinea F 1998 Phys. Rev. B 589212

[44] Stagarescu C B et al 2000 Phys. Rev. B 619233

[45] Askerov B M, Kuliev B I and Steinshreiber V Ya 1983 Fiz. Tekh. Poluprov. 171701

[46] Lewis S P, Allen P B and Sasaki T 1997 Phys. Rev. B 5510253

[47] Millis A, Mueller R and Shraiman B 1996 Phys. Rev. B 545389 